\title{
DES EUVRES DE GUERRE AUX OFFICES NATIONAUX : L'ÉVOLUTION DE LA PRISE EN CHARGE DES VEUVES DE GUERRE (FRANCE, 1914-1924)
}

\author{
Peggy Bette
}

Comité d'histoire de la sécurité sociale | « Revue d'histoire de la protection sociale » 2016/1 Nº | pages 68 à 87

ISSN 1969-9123

DOI 10.3917/rhps.009.0068

\section{Article disponible en ligne à l'adresse :}

https://www.cairn.info/revue-d-histoire-de-la-protection-sociale-2016-1-page-68.htm

Distribution électronique Cairn.info pour Comité d'histoire de la sécurité sociale.

(C) Comité d'histoire de la sécurité sociale. Tous droits réservés pour tous pays.

La reproduction ou représentation de cet article, notamment par photocopie, n'est autorisée que dans les limites des conditions générales d'utilisation du site ou, le cas échéant, des conditions générales de la licence souscrite par votre établissement. Toute autre reproduction ou représentation, en tout ou partie, sous quelque forme et de quelque manière que ce soit, est interdite sauf accord préalable et écrit de l'éditeur, en dehors des cas prévus par la législation en vigueur en France. Il est précisé que son stockage dans une base de données est également interdit. 
Des cuvres de guerre aux offices nationaux:

l'évolution de la prise en charge des veuves de guerre (France, 1914-1924)

Résumé:

Les institutions de protection sociale en charge des victimes de guerre connaissent, au cours du premier conflit mondial, des bouleversements structurels importants. Les associations philanthropiques traditionnelles se voient doublées, dès les premiers mois des hostilités, par l'apparition de centaines œuvres de guerre, tandis qu'à partir de 1916, l'État s'implique davantage en cherchant à prendre progressivement le contrôle sur ces œuvres de guerre et en mettant en place des offices nationaux (organismes semi-publics destinés spécifiquement aux victimes de guerre). Cet article, en comparant la manière dont les œuvres de guerre d'une part, et les offices nationaux d'autre part, prennent en compte les veuves, tend à évaluer dans quelle mesure l'étatisation croissante de la protection sociale suscitée par la guerre a été favorable aux femmes.

Mots-clés: femmes, genre, œuvres philanthropiques, ONAC, veuvage, victimes de guerre.

\section{From War Charities to National Offices for Veterans and Victims of War: The Development of Support for War Widows (France, 1914-1924) \\ Abstract:}

During the First World War, the welfare institutions in charge of assisting war victims underwent major structural change. In addition to traditional charitable organisations, the first months of the war saw the emergence of hundreds of war charities. From 1916 onwards, the state became more involved, seeking to take control over the war charities and by setting up national offices (semipublic bodies whose remit was specifically war victims). This paper compares the assistance for war widows provided by the war charities and by the national offices in an attempt to assess whether the increase in state control over welfare triggered by the war benefited women.

Keywords : charities, gender, National Office for Veterans and Victims of War, widowhood, war victims, women. 


\section{Des œuvres de querre aux offices nationaux :}

L'évolution de ca prise en charge des veuves de guerre (France, 1914-1924)

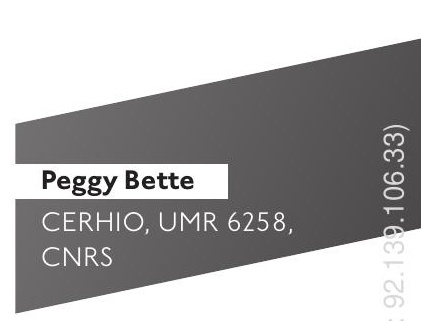

L es institutions de protection sociale en charge des victimes de guerre connaissent, du fait et au cours du premier conflit mondial, des bouleversements structurels majeurs et durables. Les œuvres philanthropiques d'avant-guerre, quoique secondées par les centaines d'œuvres de guerre qui ont émergé spontanément au cours des premiers mois des hostilités, se trouvent vite débordées par la masse des victimes à secourir. Le législateur, mû par des principes récents (solidarité) ou nouveaux (réparation), participe à la création d'administrations paritaires spécialement dédiées aux victimes de guerre : l'Office national des mutilés et réformés (ONMR) en 1916, l'Office national des pupilles de la Nation (ONPN) en 1917 et dix ans plus tard, l'Office national des anciens combattants (ONAC) en 1926. Ceux-ci fusionnent en 1946 pour former l'Office national des anciens combattants et victimes de guerre (ONACVG). La création de ces offices nationaux est considérée comme un jalon important dans le processus d'étatisation de la protection sociale'.

L'objet de cet article est de relire ce processus à l'aune du genre, en procédant à une comparaison de la situation faite aux veuves de guerre par les œuvres de guerre et les offices nationaux entre 1914 et 1924 - période de la guerre et de l'après-guerre qui correspond à l'émergence et à la coexistence de ces deux

1 Prost Antoine, Les Anciens combattants et la société française, tome 2 : Sociologie, Paris, FNSP, 1977, p. 215-235; Jackson
Harriet, «L'impact de la guerre 14-18 sur La protection sociale», in Gueslin André et Guillaume Pierre, De la charité médiévale à la
Sécurité sociale, Paris, Les Éditions ouvrières, 1992, p. 117-122. 
types d'institutions, aux principes et aux structures différentes. Le passage de la prise en charge des victimes de guerre par des organismes caritatifs à une prise en charge de ces mêmes victimes par des institutions semi-publiques a-t-il été favorable aux veuves de guerre en tant que femmes? Ont-elles été plus directement concernées par les actions sociales mises en place par les offices que par celles des œuvres ou, au contraire, plus délaissées? Quelles conceptions des femmes président à la nature et aux critères d'attribution des aides prodiguées par les unes et les autres ? En la matière, peut-on parler de rupture ou de continuité entre les manières de procéder des deux types d'institutions, entre la période de la guerre et celle de l'après-guerre?

Ce faisant, cette réflexion s'inscrit dans une problématique plus large : les impacts de la guerre sur la protection sociale française ont-ils bénéficié aux femmes ? Cette question, à la croisée des enjeux de l'histoire de la guerre, du genre et de la protection sociale, n'a pas été pleinement traitée par l'historiographie. La transition, favorisée par le premier conflit mondial, d'un système de prévoyance basé principalement sur les œuvres de bienfaisance et les mutuelles corporatives à un système supervisé par l'État a en effet rarement fait l'objet d'une approche genrée, et ce, alors même qu'historien.ne.s, sociologues et juristes ont déjà réfléchi aussi bien au caractère émancipateur des guerres sur la condition des femmes et les rapports de genre ${ }^{2}$ qu'à la dimension genrée des politiques sociales ${ }^{3}$ et des institutions sanitaires et sociales ${ }^{4}$. Si une telle approche a pu être esquissée pour l'étude de certaines politiques sociales, ainsi la réforme des pensions de guerre de $1919^{5}$, elle ne l'a pas été pour ce qui concerne les institutions et les changements structurels provoqués par les hostilités.

2 Higonnet Margaret et alii (dir.), Behind the Lines. Gender and the Two World Wars, New HavenLondon, Yale University Press, 1987 ; Thébaud Françoise, Les femmes au temps de la guerre de 14, Paris, Payot \& Rivages, 2013 (1986), notamment la conclusion de la deuxième édition (p. 419 et sq.) ; Capdevila Luc, Rouquet François, Virgili Fabrice et Voldman Danièle, Sexes, genre et guerre (France, 1914-1945), Paris, Payot, 2010 ( 1 éd. sous le titre Hommes et fermes dans la France en guerre (1914-1945), 2003).

3 Auslander Leora et ZancariniFournel Michelle, Différences des sexes et protection sociale (XIX ${ }^{e}$ $X X^{e}$ siècles), Saint-Denis, Presses universitaires de Vincennes, 1995 ; Bock Gisela, «Pauvreté féminine, droits des mères et Étatsprovidence », in Duby Georges et Perrot Michelle (dir.), Histoire des femmes en Occident, tome 5 , Thébaud Françoise (dir.), Le $X X^{e}$ siècle, Paris, Plon, 1992, p. 381409 ; Pedersen Susan, Family, Dependence and the Origins of the Welfare State. Britain and France, 1914-1945, Cambridge, Cambridge University Press, 1993, Sainsbury Diane, « Les droits sociaux des femmes et des hommes. Les dimensions du genre dans les États-providences », in Carver Terrel et alii, Genre et politique: débats et perspectives, Paris, Gallimard, 2000, p. 233-280.

4 Diebolt Evelyne, Les associations face aux institutions. Les femmes dans l'action sanitaire, sociale et culturelle, 1900-1965, thèse d'État, Université Paris VII, 1993 ; Belliard Corinne, L'émancipation des femmes à l'épreuve de la philanthropie, Paris, Éditions L'Harmattan, Paris, 2009 ; Fagnani Jeanne et
Thibault Florence (dir.), « Genre et protection sociale », Revue française des affaires sociales, $2012 / 2$.

5 Kniebiehler Yvonne, « De la veuve à la femme chef de famille (XXe siècle) ", in Souriau Christiane, Les femmes et l'argent, Aix-enProvence, CEFUP, 1986, p. 37-44 ; Lomas Janis, « "Delicate Duties": Issues of Class and Respectability in Government Policy towards the Wives and Widows of British Soldiers in the Era of the Great War », Women's History Review, vol. 9, nI, 2000, p. 123-147 ; Petit Stéphanie, «La pension de veuve de guerre de 14-18 : une pension de fidélité ? ", in Morin-Rotureau Evelyne (dir.), 1914-1918: Combats de femmes. Les femmes, pilier de l'effort de guerre, Paris, Autrement, 2004, p. 115-133; Pedersen Susan, Family, Dependence and the Origins of the Welfare 
L'analyse, menée à partir des statuts, bulletins, demandes de subventions des principales œuvres de guerre, ainsi qu'à partir des procès-verbaux des différents conseils et comités de l'ONMR et de plusieurs périodiques d'associations d'anciens combattants et victimes de guerre ${ }^{6}$, portera d'abord sur les œuvres de guerre, qui assument la majorité de l'assistance aux veuves jusqu'en 1919, puis sur l'ONMR, qui ne prend réellement le relai des œuvres qu'après la guerre.

\section{Les veuves prises en compte par les ouvres de guerre : des mères d'orphelin.e.s assisté.e.s (1914-1919)}

En 1914-1915, les œuvres dominent le terrain (les instances publiques ne participent qu'à la distribution des pensions et autres menues aides publiques) et sont soumises à un contrôle étatique lâche. Leur création, fréquemment dans le cadre juridique de la loi 1901 sur les associations, ne nécessite qu'une simple déclaration en préfecture. Quant à la provenance et à la gestion de leurs fonds, à l'origine libres de toute surveillance, elles restent du ressort du privé - même après la création en juin 1915, sur la demande du ministère, d'un comité de répartition pour garantir une redistribution équitable des fonds recueillis lors des collectes nationales. Ce comité en effet, rapidement renommé Comité de secours national (et dit aussi comité Appell en référence au nom de son fondateur, l'académicien Paul Appell), est une association loi 1901 et n'est composé que de représentants des diverses œuvres philanthropiques ${ }^{7}$. Les années 19161917 constituent un tournant juridique et institutionnel en la matière. Elles sont marquées, d'une part, par le vote de la loi du 30 mars 1916 qui impose l'obtention d'une autorisation pour toute nouvelle création d'œuvre de guerre et oblige l'ensemble des œuvres à justifier de l'argent dépensé8. Elles correspondent, d'autre part, à la création de l'Office national des mutilés et réformés (ONMR), bientôt suivie par celle de l'Office national des pupilles de la Nation en 1917, dont les actions concurrencent les initiatives des œuvres envers ces victimes de guerre. Jusqu'à la fin du conflit cependant, institutions privées et institutions semi-publiques coexistent sans que les unes n'aient un contrôle direct sur les autres. Ce n'est qu'à partir de 1919 que les œuvres de guerre commencent à marquer le pas face à un État de plus en plus intrusif dans l'assistance aux victimes de guerre.

Le monde de la bienfaisance ne néglige pas les veuves de guerre, leur proposant, sur tout le territoire, des soutiens nombreux et diversifiés. Cependant, comparativement aux autres victimes de guerre et au regard des secours proposés, les veuves restent des assistées secondaires et subordonnées.

State... op. cit.

6 Ces périodiques sont principalement Le Journal des mutilés et réformés et Les Cahiers de l'Union fédérale.

7 Comité central de secours aux orphelins, veuves et ascendants des militaires morts pour la France (Paris), Une page d'histoire de la Grande guerre : l'œuvre accomplie par le Comité central de secours aux orphelins, du 21 juin 1915 au 30 juin 1924, s.l.n.d., In-8 $8^{\circ}, 46$ p. 8 Faron Olivier, Les enfants du deuil : orphelins et pupilles de la Première Guerre mondiale, 19141941, Paris, La Découverte, 200I, p. 51 ; Thébaud Françoise,

Les femmes au temps de la guerre de 14, op. cit., p. 157 


\section{Des ouvres nombreuses et accessibles aux veuves}

La Première Guerre mondiale, par le nombre massif de ses morts et disparus, provoque le développement sans précédent d'œuvres philanthropiques nouvelles en faveur des proches des combattants défunts ou disparus. Jamais auparavant les veuves de guerre n'ont fait l'objet d'une attention aussi forte de la part du monde privé caritatif. En 1914, à la veille du déclenchement des hostilités, les seules organisations qui œuvrent spécifiquement en faveur des veuves de guerre sont peu nombreuses et généralement corporatives, à l'image de la Société spéciale de secours pour les veuves et les orphelins du génie, fondée en $185^{9}$, ou encore la Société de prévoyance pour les veuves et les orphelins des officiers de l'administration du service de l'artillerie et des officiers d'administration contrôleurs, reconnue d'utilité publique en $1874^{10}$.

Il est possible d'esquisser un panorama assez précis des œuvres destinées aux veuves de guerre à partir de l'état statistique des allocations réparties par le comité central de secours. À l'origine, cet organisme est mis en place, à la demande du ministère de l'Intérieur, pour répartir au prorata des victimes secourues par chaque œuvre les fonds récoltés lors de la première journée nationale des orphelins du 27 juin 1915 - afin que les recettes ne restent pas au seul profit de l'Orphelinat des armées, association à l'initiative de cette journée. Par la suite, le comité est de nouveau sollicité, non seulement pour assurer la redistribution des recettes acquises lors des autres journées de collecte nationale en faveur des victimes de guerre, mais aussi pour garantir la répartition équitable des subventions des ministères de l'Intérieur et de l'Instruction publique dédiées d'abord exclusivement aux orphelins puis, à partir de 1917, aux veuves et aux ascendants de guerre". L'état statistique des allocations réparties par le comité atteste que 313 œuvres au moins se sont consacrées aux veuves, orphelin.e.s et ascendant.e.s ${ }^{12}$. En raison de l'intérêt financier que représente l'affiliation au comité (sans elle, une œuvre ne peut toucher sa part des dons), il est très probable que ces associations constituent la très grande majorité des œuvres qui leur sont destinées.

L'état statistique permet, de surcroît, d'estimer précisément l'activité déployée par chacune de ces œuvres et donc de distinguer les plus importantes. Se référer aux sommes versées est d'autant plus fiable que l'usage des fonds est extrêmement contrôlé par le comité. Les demandes de subvention, admises après

9 Bibliothèque de documentation internationale et contemporaine (BDIC),

O pièce 15386, annuaires de 1916, 1917, 1918 et 1919 de la Société spéciale de secours pour les veuves et les orphelins du génie, fondée en 1858, reconnue comme établissement d'utilité publique par décret du 7 avril 1866, à Paris (7) 10 BDIC, O pièce 14218, Société de prévoyance pour les veuves et les orphelins des officiers de l'administration du service de l'artillerie et des officiers de l'administration contrôleurs d'armes, reconnue comme établissement d'utilité publique par décret du 18 avril 1874, compterendu de l'assemblée générale annuelle tenue le 3 août 1919. II BDIC, O pièce 130I3, Comité central de secours aux orphelins, Une page d'histoire de la Grande
Guerre : l'œuvre accomplie par le Comité central de secours aux orphelins, du 21 juin 1915 au 30 juin 1924.

12 BDIC, O pièce 130I3, état statistique des allocations réparties par le Comité central de secours, dans Une page d'histoire de la Grande Guerre... op.cit. 
enquête, ne sont renouvelées qu'après justification de l'emploi des subventions précédentes et, en vue d'éviter les doubles emplois (autrement dit que deux associations touchent des subventions pour une même victime), les familles secourues doivent remplir une fiche nominative qui précise l'œuvre par laquelle la subvention a été demandée ${ }^{13}$. Parmi les 313 œuvres affiliées au comité, quatorze se distinguent par la somme des subventions dont elles ont bénéficié entre 1915 et 1924, somme toujours supérieure à 700 ooo francs et pouvant s'élever jusqu'à 2800 ooo francs - alors que pour les autres œuvres, elle n'excède jamais 560 ooo francs.

\section{Principales cuvres destinées aux veuves, orphelins et ascendants de la guerre entre 1915 et 1924}

\begin{tabular}{|c|c|}
\hline Titre et adresse de l'œuvre & $\begin{array}{c}\text { Fonds attribués à cette œuvre } \\
\text { au prorata du nombre } \\
\text { des victimes secourues }\end{array}$ \\
\hline $\begin{array}{l}\text { CEuvre des Bons Enfants } \\
\text { 21, rue des Bons Enfants, à Paris }\end{array}$ & 2831 190,oo francs \\
\hline $\begin{array}{l}\text { Fédération nationale des coopératives } \\
\text { de consommation } \\
85 \text {, rue Charlot, à Paris }\end{array}$ & 2446948, oo francs \\
\hline $\begin{array}{l}\text { Association d'aide aux veuves de militaires } \\
\text { de la Grande Guerre } \\
\text { 39, rue du Général Foy, à Paris }\end{array}$ & 1790204,50 francs \\
\hline $\begin{array}{l}\text { Euvre des pupilles de l'école publique } \\
\text { de la Seine } \\
\text { 46, rue Saint Jacques, à Paris }\end{array}$ & 1653909,25 francs \\
\hline $\begin{array}{l}\text { Alliance catholique savoisienne } \\
\text { 14, rue François Miron, à Paris }\end{array}$ & 1546830 ,oo francs \\
\hline $\begin{array}{l}\text { Euvre de protection en faveur des femmes } \\
\text { et des enfants victimes de la guerre } \\
\text { 188, faubourg Saint-Martin, à Paris }\end{array}$ & 1199190,00 francs \\
\hline $\begin{array}{l}\text { Union des familles françaises et alliées } \\
\text { 29, rue Tronchet, à Paris }\end{array}$ & 1153247,15 francs \\
\hline $\begin{array}{l}\text { Société « La Bretagne» } \\
74, \text { rue de Sèvres, à Paris }\end{array}$ & 893618, oo francs \\
\hline $\begin{array}{l}\text { Action sociale en Seine-et-Oise } \\
\text { 9, rue d'Anjou, à Versailles }\end{array}$ & 868 oo6,oo francs \\
\hline $\begin{array}{l}\text { Orphelinat des armées } \\
\text { 21, rue Jacob, à Paris }\end{array}$ & 811353,65 francs \\
\hline $\begin{array}{l}\text { Association française pour la protection } \\
\text { des familles des morts pour la patrie } \\
\text { 8o, rue de l'Université, à Paris }\end{array}$ & 810 319,oo francs \\
\hline
\end{tabular}

13 BDIC, O pièce 13013, Comité central de secours aux orphelins, veuves et ascendants des (article 7 et 8 du règlement pour l'attribution des subventions). 


\begin{tabular}{|l|c|}
\hline \multicolumn{1}{|c|}{ Titre et adresse de l'œuvre } & $\begin{array}{c}\text { Fonds attribués à cette œuvre } \\
\text { au prorata du nombre } \\
\text { des victimes secourues }\end{array}$ \\
\hline $\begin{array}{l}\text { Orphelinats de la fédération des tabacs } \\
\text { Manufactures des tabacs, à } \\
\text { Issy-les-Moulineaux }\end{array}$ & 799594, oo francs \\
\hline $\begin{array}{l}\text { Union aveyronnaise (Lozère) } \\
61, \text { rue d'Anjou, à Paris }\end{array}$ & 749526,00 francs \\
\hline $\begin{array}{l}\text { Comité girondin de protection des orphelins } \\
\text { de l'armée } \\
\text { 9, rue de Cheverus, à Bordeaux }\end{array}$ & 701205,65 francs \\
\hline
\end{tabular}

Source: Comité central de secours aux orphelins, veuves et ascendants des militaires morts pour la France (Paris), Une page d'histoire de la Grande Guerre... op. cit., s.l.n.d., In- $8^{\circ}, 46$ p.

Rares ont dû être les veuves nécessiteuses qui n'ont pu recourir à la bienfaisance privée faute d'œuvres à proximité de leur domicile, non seulement en raison de leur abondance, mais aussi de leur présence très ramifiée sur le territoire, qui repose sur des structures et des réseaux antérieurs à la guerre et avec lesquels les veuves sont souvent en contact. Parmi eux, l’Église tient le premier rang. Le monde catholique est en effet l'un des premiers à se mobiliser, soutenant notamment la création, en novembre 1914, de l'Association nationale pour la protection des veuves et des orphelins, dite plus communément Les Bons Enfants (en référence au nom de la rue où se trouve son siège). Son fondateur, l'avocat à la Cour et vice-président du conseil général de la Seine, Ambroise Rendu, bénéficie de l'appui de l'archevêque de Paris, le cardinal Amette, et confie la présidence du conseil d'administration de l'œuvre à la duchesse d'Uzès, grande figure de la philanthropie chrétienne et du féminisme réformiste ${ }^{14}$. Cette appartenance catholique se retrouve sur le terrain. Les Bons Enfants fonctionnent grâce à l'entremise de sociétés et confréries catholiques nées au XIX'e siècle, telle la Société de Saint-Vincent-de Paul (qui assure les enquêtes dans Paris et sa banlieue) ${ }^{15}$ ou encore les Dames du Calvaire (qui sont chargées, avec d'autres dames visiteuses, de la distribution des aides pécuniaires). Ils recourent également aux réseaux des paroisses et trouvent dans la personne des évêques et curés, ainsi que dans celle des instituteurs et institutrices des écoles libres, des intermédiaires indispensables pour s'implanter dans les campagnes reculées ${ }^{16}$.

La société militaire s'engage elle aussi en faveur des veuves des combattants, principalement via l'Association d'aide aux veuves de militaires de la Grande Guerre, créée en juin 1915 à l'initiative de la baronne Lejeune, née princesse Marguerite Murat, en mémoire de son époux Jules Marie Edgard Lejeune, capitaine du $5^{\mathrm{e}}$ régiment de cuirassier, mort en novembre 1914 des suites de ses

14 Bard Christine, Les filles de Marianne. Histoire des féminismes, 1914-1940, Paris, Fayard, 1995, p. 242 et 273 .

$15 \mathrm{BDIC}, 8^{\circ} \mathrm{P} 815$, Bulletin de l'Association nationale de la protection des veuves et des orphelins de guerre, mars 1916, p. IO, (note I).

16 Ibid., p. 12-13; voir aussi La Semaine religieuse du diocèse de Mende, par exemple l'article «Orphelins de la guerre, élèves des écoles libres », du vendredi 21 avril 1916, p. 193-194; ou encore l'encart « Avis » paru dans le numéro du vendredi 19 mai 1916, p. 242-243. 
blessures ${ }^{17}$. L'œuvre, dont le conseil d'administration est composé de personnalités militaires et/ou de la noblesse bonapartiste, calque son organisation et son développement sur la structure institutionnelle de l'armée - centralisée dans la capitale, avec des antennes dans chaque région militaire de province. Elle possède à Paris un secrétariat général, appelé initialement " comité des dames ", chargé spécialement, après enquête, de rechercher des emplois, des situations, du travail et de proposer, s'il y a lieu, des secours en argent. Des comités similaires sont placés dans chaque région de corps d'armée (quand il s'agit de l'armée de terre) et dans chaque arrondissement maritime (pour la marine). Chaque comité est lui-même divisé en sous-comités et groupements. Le développement de l'œuvre est rapide, passant de cinq comités à la fin de l'année 1915 à quinze deux ans plus tard, auxquels il faut ajouter, à partir de 1918, celui de Lille (occupée pendant les hostilités) ${ }^{18}$. Ce développement a été conditionné par une convention passée avec les responsables de l'œuvre des Bons Enfants, les deux associations ayant constaté, lors de l'attribution des fonds de la première journée nationale des orphelins de guerre en 1915, qu'elles touchaient le même type de population et risquaient donc de faire souvent double emploi's. Ainsi, là où est l'une, l'autre n'est pas. En 1935, lors de sa dissolution, l'Association d'aide aux veuves de militaires de la Grande Guerre affirme avoir secouru 40 ooo veuves et 6 o ooo orphelin.e.s pour l'ensemble de la France.

Face à ces deux réseaux de la sphère catholique se déploie celui de la communauté éducative dite "laïque », qui se mobilise en faveur des veuves de guerre par le biais de ses œuvres des pupilles de l'école publique, coordonnées à partir de 1917 par la Fédération nationale des associations départementales des pupilles de l'école publique. Présidée par le ministre de tendance radicalsocialiste Léon Bourgeois, l'œuvre est à l'origine destinée aux seuls orphelins et orphelines d'enseignants décédés à la guerre, puis s'adresse rapidement à l'ensemble des enfants scolarisés privés de père par la guerre. La première des œuvres des pupilles de l'école publique est fondée à Paris, à l'initiative d'universitaires parisiens officiant dans tous les cadres de l'enseignement public (primaire, secondaire et supérieur) et parmi lesquels se trouvent des personnalités comme Émile Durkheim ou Pauline Kergomard. Sise rue Gay-Lussac, dans l'enceinte du musée pédagogique, l'œuvre cherche, dès la fin 1915, à s'étendre en province. Un « comité d'initiative » encourage, par le biais de circulaires, la

17 BDIC, O pièce 19356, Une cuvre de guerre : Association d'aide aux veuves de militaires de la Grande Guerre, 1915-1935, Notice historique, Dijon, 1935. A propos de la baronne Lejeune, princesse Marguerite Murat, voir le dossier de légion d'honneur consultable en ligne sur le site des Archives nationales, base LEONORE. À propos de son époux, Jules Marie Edgard (né le 31 janvier 1881), voir la fiche « mémoire des hommes » mise en ligne sur le site mémoires des hommes du SGA http://www.memoiresdeshommes. sga.defense.gouv.fr

18 BDIC, O pièce 19356, Une cuvre de guerre : Association d'aide aux veuves de militaires de la Grande Guerre, 1915-1935, voir supra. Le fonctionnement et le développement de l'œuvre est également décrit dans l'ouvrage de l'avocate Suzanne Grinberg, Les droits des veuves et des orphelins de guerre, Paris, Albin Michel, 1916. $19 \mathrm{BDIC}, \mathrm{O}$ pièce $8^{\circ} \mathrm{P} 815$, Bulletin de l'Association nationale pour la protection des veuves et des orphelins de la guerre de 1914, mars 1916, p. 34-35 (l'Association d'aide aux veuves de militaires $y$ est désignée sous la dénomination "l'œuvre de la rue Vézelay », en référence à l'adresse de son siège sociale en 1916); BDIC, O pièce 19356, Une œuvre de guerre. Association d'aide aux veuves de militaires de la Grande Guerre, op. cit, p. 56. 
création d'œuvres similaires dans chaque département, dont la composition et le fonctionnement sont appelés, quoiqu'indépendants, à être très analogues d'un département à l'autre pour assurer l'unité de l'œuvre au plan national. Les inspecteurs d'académie en deviennent les présidents, tandis que les principaux des collèges, les directrices des cours secondaires, les directeurs et directrices des écoles normales et les inspecteurs du primaire sont désignés membres de droit du conseil d'administration ${ }^{20}$. Des comités cantonaux sont également créés, composés d'instituteurs, d'institutrices et d'enseignant.e.s du secondaire qui se font agents de liaison et de renseignements entre l'œuvre et les familles secourues. Quant aux élèves, ils sont directement sollicités en étant incités à apporter mensuellement une pièce (ou plusieurs selon le niveau de fortune de leur famille) pour aider leurs camarades orphelin.e.s de guerre ${ }^{21}$. Cette « obole des écoliers », nommée également « sou des écoliers », sans être l'apport le plus rémunérateur de l'œuvre, constitue un outil de sensibilisation majeur pour la cause des orphelins et de leur famille. En 1918, l'œuvre des pupilles rayonne sur quasiment tout le territoire métropolitain avec 85 associations, prenant en charge presque 150 ooo orphelin.e.s (ce qui correspond en moyenne à 1700 par département) dont les mères, veuves de guerre, se trouvent financièrement et moralement soutenues ${ }^{22}$.

\section{Les veuves, des victimes secondaires et subordonnées}

En dépit de leur abondance et de leur diversité, ces actions caritatives s'adressent rarement, voire jamais, aux seules veuves de guerre ; elles restent, comme au XIX ${ }^{\mathrm{e}}$ siècle, étroitement liées à celles des orphelins. Ce phénomène est perceptible à travers les intitulés des œuvres. Parmi les quatorze les plus importantes soutenues par le comité Appell, une seule - l'Association d'aide aux veuves de militaires de la Grande Guerre - s'adresse spécifiquement, par son intitulé, aux veuves. Toutes les autres visent soit la seule population des orphelins et orphelines (ainsi l'Orphelinat des armées, l'œuvre des pupilles de l'école publique de la Seine, l'Orphelinat de la fédération des tabacs ou encore le Comité girondin de la protection des orphelins des armées), soit le binôme classique de la veuve et de l'orphelin (tels Les Bons Enfants, ou l'CEuvre de protection en faveur des femmes et des enfants victimes de guerre), soit enfin l'ensemble des membres des familles éprouvées (à l'instar de l'Union des familles françaises et alliées, de l'Association nationale pour la protection des familles des morts pour la patrie, de l'Union catholique savoisienne, de la société « La Bretagne » ou encore de l'Union aveyronnaise).

Cette fusion/confusion, dans les intitulés, de la cause des veuves avec celle des orphelins ou des familles éprouvées dans leur entier, n'est pas liée à l'ampleur

$20 \mathrm{BNF}, 8^{\circ} \mathrm{JO} 2866 \mathrm{I}$, Bulletin de l'CEuvre des pupilles de l'école publique de Seine-et-Marne, 1916/1920, $\mathrm{n}^{\circ}$ ।.

21 BNF, Pièce $8^{\circ} \mathrm{R} 14207$, OEuvre départementale des pupilles de l'école publique du Doubs. Orphelins de guerre, Besançon, La Solidarité, Imprimerie Coopérative, 6 et 8 rue Gambetta, 1916, ( 16 p.), p. 7 ; BNF, 8० JO 2866I, Bulletin de l'CEuvre des pupilles de l'école publique de Seine-et-Marne, 1916/1920, n I, p. 13 ; Faron Olivier, Les enfants du deuil, op. cit., p. 73 22 Faron Olivier, Les enfants $d u$ deuil, op. cit., p. 72. 
du rayonnement des associations concernées, puisqu'on la retrouve au niveau d'associations plus modestes. Là aussi, une seule œuvre (sur les 299 ayant reçu moins de 560 ooo francs de la part du comité national de secours entre 1915 et 1924) s'adresse, par sa dénomination, uniquement aux veuves de guerre. Il s'agit de l'Assistance mutuelle aux veuves de guerre ${ }^{23}$, mutuelle (d'obédience catholique) fondée par l'académicien Frédéric Masson en mai $1915^{24}$. En réalité, à y regarder de plus près, cette œuvre ne s'occupe en fait pas exclusivement des veuves de guerre : la cause des orphelins fait partie de ses missions principales. De fait, l'insécabilité de la cause des veuves avec celle des orphelins n'est pas que formelle. Elle est également effective dans la nature des aides prodiguées : aucune forme d'assistance n'est proposée aux veuves sans l'être aussi aux orphelins. Les bénéficiaires des aides médicales et la prise en charge de l'hospitalisation en sont un premier exemple. Les consultations médicales gratuites proposées aux veuves par l'Euvre de protection des femmes et des enfants, ou par l'Association pour la protection des familles des morts pour la patrie, se déroulent dans un dispensaire ouvert à toute la famille ${ }^{25}$. De même, la maison de repos «Les Terrasses » a été instituée à Niort en 1919 par l'Association d'aide aux veuves des militaires de la Grande Guerre pour « hospitaliser des veuves et des orphelins déprimés ou ayant besoin de repos ${ }^{\mathbf{2}}{ }^{6}$.

Le même phénomène concerne les aides au placement professionnel, pensées autant pour les veuves que pour les orphelin.e.s en âge de travailler - et ce, même lorsque l'œuvre se destine principalement aux veuves par son intitulé. Fin 1915, la commission de travail des Bons Enfants a permis quarante-deux placements définitifs de veuves ou d'orphelins, seize placements temporaires, a satisfait vingt-deux demandes de travail à domicile et donné quatre machines à coudre ${ }^{\mathbf{2 7}}$. De même, les rapports de l'association d'aide aux veuves de la Grande Guerre lors de l'assemblée générale de 1918 précisent que « des femmes et des enfants ont été placés en grand nombre », dont dix dans les bureaux militaires par le sous-comité du Maine-et-Loire ${ }^{\mathbf{2 8}}$.

Cette confusion veuves/orphelins apparaît également dans les critères d'attribution des aides. Il est en effet très courant que les œuvres conditionnent leur soutien aux veuves à la présence d'orphelins et aux seuls besoins de ces

23 OEuvre n61 de l'état statistique établi dans Comité central de secours aux orphelins, veuves et ascendants des militaires morts pour la France (Paris), Une page d'histoire de la Grande Guerre..., op. cit.

24 CAC 200502063 , ONMR, procès-verbal du conseil de perfectionnement, demandes de subventions, « Mutuelle des veuves de guerre », séance du 22 juillet 1925 .

25 CAC 200502063 , ONMR, procès-verbal du conseil de perfectionnement, demandes de subventions, « OEuvre de protection en faveur des femmes et des enfants victimes de guerre ", séance du 24 septembre 1924 ; ibid., séance du 23 septembre 1925 ; BDIC, O pièce 14152, Association nationale française pour la protection des familles des morts pour la France.

26 CAC 200502062 , ONMR, procès-verbal du conseil de perfectionnement, demandes de subventions, «Association d'aide aux veuves de la Grande Guerre », séance du o9 décembre 1921. Le soulignement du « et » est de mon fait.

27 BDIC, $8^{\circ} \mathrm{P} 815$, Bulletin de l'Association nationale pour la protection des veuves et des orphelins de la guerre de 1914, mars 1916, p. II-12.

28 BDIC, O pièce I4I5O,

Association d'aide aux veuves de militaires de la Grande Guerre (armées de terre et de mer), assemblée générale du 2 février 1918, rapport du président et des directrices de section. Pour la $9^{\mathrm{e}}$ région. 
derniers. Ceci est manifeste pour les secours pécuniaires, principale forme de soutien proposée par les œuvres et couramment distribués en fonction du nombre d'enfants. Les veuves, mères des orphelins assistés, ne sont considérées que comme des récipiendaires et des gestionnaires de la somme versée. Fin décembre 1915, l'œuvre des Bons Enfants s'occupe à Paris et en région parisienne de 1061 orphelins et de leurs mères, auxquelles ils remettent tous les mois à domicile un secours de 10 francs par enfant ${ }^{29}$. Face aux demandes croissantes, l'œuvre des pupilles de l'école publique de la Seine-et-Marne privilégie, à compter de juin 1917, les familles composées de trois enfants âgés de moins de 14 ans, à qui elle verse mensuellement 5 francs par enfant ${ }^{30}$. Seule, peut-être, l'assistance juridique a pu être dispensée aux veuves sans référence à leur rôle de mère. Encore faut-il remarquer que cette aide est souvent proposée dans l'intérêt direct des orphelins, dont il faut instruire les dossiers de demande de secours par l'intermédiaire de leurs mères.

En somme, les veuves, lorsqu'elles sont soutenues par les œuvres de bienfaisance, le sont soit au nom de leurs enfants (secours pécuniaires), soit dans l'intérêt de ces enfants. Il s'agit de garantir à ces derniers un bon développement en assurant à leur mère, une bonne santé, un revenu professionnel ou une capacité à demander des aides publiques. Les œuvres dans leur ensemble, et plus particulièrement les œuvres catholiques, placent toujours, pour reprendre l'expression d'Olivier Faron, «la famille par-dessus tout »- famille dont il faut sauvegarder le maintien et la cohésion afin d'assurer l'avenir de la patrie. Ainsi les veuves ne sont-elles jamais reconnues isolément, ne sont prises en considération qu'en tant que mères et dans la mesure où elles peuvent assurer l'intégrité de la cellule familiale ${ }^{31}$.

Ces objectifs moraux soulignent un autre lien de subordination subi par les veuves : celui que développent les animatrices et animateurs des œuvres envers elles. Ces veuves secourues restent, en effet, souvent cantonnées dans la posture passive de l'assistée. Peu d'œuvres fonctionnent selon le principe de l'entraide et quand c'est le cas, à l'image de l'Association d'aide aux veuves de militaires de la Grande Guerre, cette entraide n'empêche pas le maintien de la hiérarchie sociale et des rapports de classes. Ce sont des veuves d'officiers qui cherchent à aider des veuves de simples soldats, suivant une posture assimilable à celles des dames patronnesses. Pour la baronne Lejeune, fondatrice et présidente de l'Association d'aide aux veuves de militaires de la Grande Guerre, l'important est moins d'assurer le secours matériel des veuves dans le besoin que de les « instruire [...] de leurs droits, de leurs devoirs et d'être dans toutes les questions juridiques ou morales leur appui et leur guide »32. À cet égard, il convient de mentionner le caractère exceptionnel de l'Association mutuelle des

$29 \mathrm{BDIC}, 8^{\circ} \mathrm{P} 815$, Bulletin de l'Association nationale pour la protection des veuves et des orphelins de la guerre de 1914, mars 1916, p. 11-12.
$30 \mathrm{BNF}, 8^{\circ} \mathrm{JO} .2866 \mathrm{I}$, Bulletin de l'oEuvre des pupilles de l'école publique de Seine-et-Marne, 1916/1920, n I, Fontainebleau, Imprimerie Hunot, I5, rue du château.

31 Faron Olivier, Les enfants du deuil, op. cit., p. 68-70. 
veuves de guerre, déjà citée, qui, comme son nom l'indique, fonctionne selon des principes mutualistes - et par conséquent, se départit du rapport hiérarchique entre aidants et aidés en vigueur dans les autres œuvres de guerre. Dans cette configuration, les veuves, qui versent chacune une cotisation pour appartenir à la mutuelle, sont des adhérentes et non des assistées. Son rayonnement et son développement restent cependant limités (la mutuelle compte 500 adhérentes en 1925), comme si un tel mode de fonctionnement ne pouvait s'étendre ou susciter des groupements analogues dans le monde caritatif. Il a, en revanche, été couramment adopté et/ou encouragé par les associations d'entraide fondées à l'initiative des victimes de guerre elles-mêmes et qui se multiplient surtout après le conflit ${ }^{33}$.

La fin de la guerre ne provoque pas la disparition des œuvres de guerre. Beaucoup perdurent jusque dans les années 1930, voire au-delà, après la Deuxième Guerre mondiale, à l'exemple des Bons Enfants dont la vitalité ne se dément pas jusque dans les années $1950^{34}$. Pour certaines mêmes, les premières années de paix sont le temps de leur émergence, à l'image de l'CEuvre d'assistance médicale aux veuves et aux orphelins des officiers de la guerre, fondée en 1919 par l'épouse du maréchal Foch dans le cadre de la Fondation de la victoire et qui, comme l'Association d'aide aux veuves des militaires de la Grande Guerre, prend ses assises dans la société militaire et plus spécifiquement auprès de la noblesse bonapartiste ${ }^{35}$. Néanmoins, leurs actions sont désormais fortement concurrencées par celles que déploient les offices nationaux et elles dépendent de plus en plus, au fur et à mesure, de ces mêmes offices, suite au changement de statut du comité Appell (devenu en 1918 un établissement public sous contrôle de l'État ${ }^{36}$ ) puis, à partir de 1922, au transfert des compétences de ce même comité aux offices nationaux. Lorsque le zo juin 1924 le comité est définitivement dissout, les offices nationaux sont devenus les seules instances à assumer l'instruction et la distribution des subventions au profit d'associations dédiées aux victimes de guerre ; parmi elles, les associations de bienfaisance doivent de plus en plus partager le terrain avec les associations d'entraide.

\section{9-1924 : les veuves prises en compte par les offices nationaux : des ayants droit à part entière?}

Les offices nationaux, quoique fondés en 1916 et 1917, ne commencent réellement à fonctionner qu'à la fin de la guerre. Quand l'Office national des mutilés et réformés (ONMR) est créé en mars 1916 pour organiser l'emploi des mutilés dans le secteur privé, il n'est qu' « un simple comité de liaison entre services de

32 BDIC, O pièce 14150 , Association d'aide aux veuves de militaires de la Grande Guerre (armées de terre et de mer), Rapport du président et des directrices de section de la $9^{\mathrm{e}}$ région (Tours).

33 Prost Antoine, Les anciens combattants et la société française, tome 1 : Histoire, Paris, FNSP, 1977, p. 47 sq. ; ibid., tome 2 : Sociologie, p. 229 sq.

34 Faron Olivier, «Aux côtés, avec, pour les pupilles de la Nation : les formes de la mobilisation en faveur des orphelins de la Première Guerre mondiale », Guerres mondiales et conflits contemporains, $\mathrm{n}^{\circ} 295$, 2002/I, p. 15-33, p. 24. $35 \mathrm{BNF}, \mathrm{JO}-8226$, Bulletin de l'œEuvre d'assistance médicale aux veuves et orphelins des officiers de la guerre, janvier 1922, $n^{\circ} 2$.

36 Faron Olivier, Les enfants $d u$ deuil, op. cit., p. 159. 
différents ministères dépourvu de moyens propres »37. La loi du 2 janvier 1918 fait évoluer son statut en le transformant «en un établissement public chargé de tout le secteur de la rééducation et du placement des mutilés »38. À la fin du conflit, cet établissement rattaché au ministère du Travail, doté de la personnalité civile et d'un budget conséquent versé par l'État, supervise tout ce qui relève du reclassement des mutilés. Il a la capacité juridique et financière de créer des écoles, de même que d'accorder des prêts ou des secours ${ }^{39}$. L'Office national des pupilles de la Nation (ONPN), prévu par la loi du 27 juillet 1917 instituant les pupilles de la Nation, nécessite un délai de mise en place et d'adaptation comparable. Si la première session du conseil supérieur de l'office a lieu moins d'un an plus tard (mars 1918), il faut attendre novembre 1918 pour que la presque totalité des comités départementaux soient en état de fonctionner encore que dans des conditions très rudimentaires et ce, parfois jusqu'au début des années $1920^{40}$. Reste que, une fois en place, l'armature des offices, dont la ramification départementale et locale n'a rien à envier à celle des œuvres de guerre, est très efficace. En contact constant avec les associations d'entraide des anciens combattants et victimes de guerre, ils parviennent à toucher une partie considérable de la population qu'ils ont pour mission de servir. Antoine Prost estime que, dans les années 1920 et 1930, « les offices nationaux et leurs comités départementaux constituent l'un des principaux réseaux d'aide sociale, le plus puissant et, à coup sûr, le mieux organisé "4

Comparativement aux invalides et aux orphelin.e.s, il semblerait que les veuves aient été plus tardivement et/ou plus indirectement prises en compte par les offices nationaux. La raison en est que, comme en témoigne leur dénomination, aucun de ces deux offices n'a, à l'origine, la mission de porter une attention spécifique à leur cas (il est à remarquer à cet égard qu'aucun office national de veuves de guerre n'a été, et ne sera jamais, fondé). L'ONMR ne commence à prendre en compte les veuves dans ses missions qu'à partir de 1919 à la suite de l'extension, par la loi du 31 mars 1919, du droit à la formation professionnelle aux femmes pensionnées ${ }^{42}$. Quant à l'ONPN, en vertu de ses missions à l'égard des orphelins de guerre et pupilles de la Nation, il considère exclusivement les veuves mères d'orphelins de guerre mineurs, ce qui n'est pas sans rappeler la position et la perception des œuvres de guerre. Néanmoins, les principes juridiques et moraux qui sont à l'origine de la création des offices, leur mode de fonctionnement novateur, de même que les programmes d'action sociale qu'ils mettent en œuvre, modifient la place réservée jusqu'ici aux veuves, tant par les œuvres caritatives que par les pouvoirs publics.

37 Prost Antoine, Les anciens combattants et la société française, tome I: Histoire, op. cit. p. 80 .

38 Ibid.p. 81.

39 Ibid.p. 21 et 81.

40 Faron Olivier, Les enfants du deuil, op. cit., p. 115-117.

41 Prost Antoine, Les anciens

combattants et la société

française, tome 2 : Sociologie, op.

cit., p. 229.

42 Art. 76 de la loi du

31 mars 1919 modifiant la législation des pensions des armées de terre et de mer en ce qui concerne les décès survenus, les blessures reçues et les maladies contractées et aggravées en service, publiée au Journal Officiel, Lois et décrets, le 2 avril 1919, p. 3389. 


\section{Des ayants droit plutôt que des assistées}

Dans le contexte d'une guerre patriotique engendrant des morts en masse, le législateur conçoit désormais la guerre comme un risque social et place les droits des victimes de guerre sous le principe de la « réparation », principe qui assimile leurs pensions non plus à un secours ou un droit (dérivé) professionnel, mais à l'indemnisation d'un préjudice subi. Partant, les victimes de guerre passent de la posture d'assistées, redevables d'œuvres qui consentent à leur porter secours, à celle d'ayants droit, créancières de la nation ; les dons d'hier sont devenus un dû ; les initiatives caritatives, une politique de solidarité nationale. La création des offices, avant même la réforme des pensions, incarne ce changement de prise en considération. Comme le souligne Harriet Jackson, leur création émane autant d'une nécessité matérielle et logistique (il fallait éviter, explique-t-elle, que l'importante population de mutilés, veuves, orphelins et ascendants de guerre ne « se retrouvent dans la misère et affluent dans les bureaux de l'assistance publique »), qu'une question de principe (le législateur jugeait l'assistance publique pas assez digne de ces ayants droit qui représentaient le sacrifice fait par les héros «morts pour la France ») ${ }^{43}$. Le personnel des offices confirme ce changement de prise en considération. Là, pas de dames patronnesses ou de veuves d'officiers venant au secours des veuves les plus modestes, ni de fonctionnaires à l'attitude distante envers les cas qui se présentent, mais une institution composée de victimes de guerre au service des victimes de guerre. L'historien Antoine Prost remarque que « de part et d'autre de la banque, on appartient à la même franc-maçonnerie des misères de la guerre. D'où une cordialité, une attention, une patience » que l'on ne trouve pas ailleurs dans l'Administration ${ }^{44}$.

Mais c'est sans doute le mode de gestion des offices qui incarne le mieux ce changement dans la prise en considération des victimes de guerre. Les offices nationaux présentent en effet l'originalité d'être des structures semi-publiques, dont le cadre est assuré par l'État et l'animation par des membres de la société civile directement concernés par la question des victimes de guerre, soit parce qu'ils leur procurent des secours (comme les responsables d'œuvres de bienfaisance), soit en étant eux-mêmes victimes (mutilés, veuves). Leur gestion est entre les mains d'instances paritaires où sont représentées l'ensemble de ces parties (d'un côté des fonctionnaires, de l'autre des philanthropes et victimes de guerre). La place de ces dernières - les victimes de guerre - croît à mesure du temps. D'abord au sein des instances de l'ONMR, où les mutilés réclament en 1920, dans le but de préserver l'autonomie de l'ONMR vis-à-vis du ministère des Pensions tout récemment créé, que la moitié des sièges des comités départementaux et la moitié de ceux du conseil de l'office national reviennent à des représentants élus de leurs associations ${ }^{45}$. Ensuite, au sein des instances de

43 Jackson Harriet, « L'impact de la guerre sur la protection sociale »... op. cit., p. 119.

44 Prost Antoine, Les anciens combattants et la société française, tome 2 : Sociologie, op. cit., p. 229.

45 Prost Antoine, Les anciens combattants et la société française, tome 1 : Histoire, op. cit., p. 81 et 82 . 
l'ONPN qui, par la nouvelle loi du 26 octobre 1922 sur les pupilles de la Nation, s'ouvrent aux représentants des groupements de mutilés, tant à l'échelle nationale que départementale et cantonale ${ }^{46}$.

Ce mode de gestion paritaire permet aux veuves de se voir représentées par certaines d'entre elles dans les instances dirigeantes des institutions qui les prennent en charge et, par conséquent, d'être directement impliquées dans le choix et les perspectives des politiques mises en place. Ce qui est rarement le cas au sein des directions des œuvres de guerre, et impossible au sein du comité de secours national, celui-ci ayant un bureau entièrement masculin ${ }^{47}$. À l'échelle nationale, certaines, telle la baronne Marguerite Lejeune (présidente-fondatrice de l'Association d'aide aux veuves des militaires de la Grande Guerre) ${ }^{48}$ ou Thérèse Casevitz (écrivaine et militante de l'Union française pour le suffrage des femmes) ${ }^{49}$, ne le font pas en vertu de leur statut de veuve de guerre, mais de leur engagement au sein d'associations philanthropiques ou féministes. Il est également à souligner, dans leur cas, que leur nomination n'a concerné que l'ONPN, dont le règlement intérieur impose, dès sa mise en place en 1918, un quota de femmes variant d'un tiers à un quart selon les conseils et commissions concernés. Toutes deux sont alors des femmes représentant les orphelins et non les veuves.

Le début des années 1920 ouvre la voie à des veuves de guerre qui présentent un tout autre profil, plus en adéquation avec la nouvelle conception des victimes de guerre. Citons, à cet égard, deux d'entre elles : Élisabeth Cassou, nommée à l'ONMR en 1922 et à l'ONPN en $1923^{50}$, et Jeanne Callarec, nommée à l'ONPN en $1923^{51}$. Aucune ne peut être assimilée à une dame patronnesse ; elles appartiennent à cette nouvelle élite féminine que constituent les enseignantes de l'instruction publique, la première étant professeure en école primaire supérieure dans les Landes (puis à Paris), la deuxième institutrice et directrice d'école dans le Finistère. Toutes deux doivent leur nomination à leur élection au conseil d'administration national de l'un des plus importants groupements combattants de l'époque, l'Union fédérale des anciens combattants et victimes de guerre (UF), dont elles sont respectivement secrétaire générale et vice-présidente des années 1920 aux années $1950^{52}$. Durant ces trente années, l'une et l'autre réunissent sous leur nom, lors des élections annuelles du conseil d'administration national de l'UF, entre $76 \%$ et $80 \%$ des suffrages. Ainsi, à la différence de la baronne Lejeune ou de Thérèse Casevitz qui ont été nommées pour leurs actions en faveur des victimes de guerre, Élisabeth Cassou et Jeanne

46 Faron Olivier, Les enfants du deuil, op. cit., p. 130.

47 Thébaud Françoise, Les femmes au temps de... op. cit., p. 151. 48 CAC, 19800035/222/29312, notice $n^{\circ} \mathrm{C}-212014$, dossier de la Légion d'honneur de la baronne Lejeune, née Princesse Marguerite Malcy Caroline Alexandrine Murat (consultable en ligne sur le site des Archives nationales, base
LEONORE).

49 Anonyme, «Mme Casevitz à l'Office national des pupilles de la Nation », La Française, 28 juillet 1923, p. 2.

50 Archives de la Grande Chancellerie de la Légion d'honneur, dossier de la Légion d'honneur d'Élisabeth Vernet (Veuve Cassou).

51 Archives nationales,
L0409057, dossier de la Légion d'honneur de Jeanne Callarec ; René Cassin, «La mère des pupilles », Cahiers de l'Union fédérale, $n^{\circ}$ 53, février 1951, p. 3. 52 Archives de l'Union fédérale, registre mentionnant la composition des bureaux successifs. 
Callarec ont été élues, non seulement pour leur engagement, mais aussi par les victimes de guerre pour les représenter. Elles tirent leur légitimité autant de leur rôle dans le mouvement associatif que de leur assise populaire.

Cela étant, les veuves de guerre sont probablement sous-représentées au sein des instances dirigeantes des offices nationaux, parce qu'elles le sont déjà au sein des représentant.e.s élu.e.s des différents groupements d'anciens combattants et de victimes de guerre, d'où proviennent la moitié des membres dirigeants des comités départementaux, puis des offices nationaux. Seulement quinze veuves ont pu être recensées parmi les membres des conseils d'administration des principales unions et fédérations nationales, dont une très grande majorité issue de l'UF (sept) ${ }^{53}$, parmi lesquelles Jeanne Callarec et Élisabeth Cassou, déjà citées, qui semblent être les seules à s'être hissées à l'échelon national des offices. Il serait néanmoins nécessaire, pour convertir cette forte probabilité en certitude, d'entreprendre la récapitulation de l'ensemble des personnalités qui ont siégé dans les divers conseils et comités des offices pendant l'entre-deux guerres et de comparer la place des veuves par rapport à la place occupée par les mutilés.

Autre spécificité de fonctionnement des offices qui provoque un changement dans la considération de ces veuves : la prise en charge de leur cas par l'Office national des mutilés et réformés. Ce rattachement institutionnel, de même que la nature des principales politiques qui leur sont dédiées (il s'agit de politiques d'emploi), manifeste une rupture dans la perception des veuves de guerre par les institutions de protection sociale. Les voilà départies de leur rôle de mère qui les associait irrémédiablement au sort de leurs enfants, pour endosser celui de travailleuses à classer ou reclasser, à l'instar des mutilés. Rupture significative, mais qui reste néanmoins limitée, et ce pour deux raisons. D'une part, parce qu'il semblerait qu'en dépit de ce transfert institutionnel, le peu de veuves de guerre siégeant dans les offices nationaux continuent à être principalement affectées à l'office des pupilles. D'autre part, parce que les politiques d'emploi mises en œuvre, par la nature des emplois proposés et les critères d'attribution des aides, montrent une certaine continuité avec les conceptions que les œuvres de guerre avaient des veuves de guerre, en raison de leur sexe.

\section{Des ayants droit femmes et mères}

Les politiques d'emploi proposées par l'ONMR en faveur des veuves de guerre se déploient en deux grands volets. L'un concerne la formation professionnelle. Depuis le vote de la loi du 31 mars 1919 réformant les pensions de guerre, et en vertu de son article 76, les veuves de guerre y ont droit, à l'instar de ce qui est proposé aux mutilés depuis 1916. L'ONMR leur offre donc la possibilité de se former ou de se reconvertir professionnellement, soit en intégrant une école

53 Journal des mutilés (1916 à 1944); archives privées de l'UF et de l'UNC; archives Marianne Rauze

(vice-présidente de l'ARAC), qui semble n'avoir jamais siégé dans aucun office. 
de rééducation, soit en suivant un apprentissage « chez un patron », soit en suivant des cours à domicile ou par correspondance. L'autre volet concerne les travailleuses indépendantes, dont l'ONMR cherche à soutenir l'installation et le développement en proposant des aides à la fondation de coopératives ouvrières de production (COP), en accordant des prêts d'honneur et des prêts professionnels, ou encore en prêtant gracieusement des machines à coudre. Au cours de la période 1920-1926 $6^{54}$, plus de 30 ooo veuves ont bénéficié de ces politiques : 608 ont suivi une formation en école, 1722 «chez un patron », quelques dizaines ont été soutenues pour fonder une COP, 734 ont obtenu un prêt d'honneur, 10 ooo environ une machine à coudre. Minime au regard du nombre total de veuves de guerre (70o ooo), le nombre des bénéficiaires est néanmoins considérable quand l'on songe qu'il s'agit de politiques innovantes et sans précédent, et qui sont poursuivies pour certaines d'entre elles au-delà de 1930 - même si c'est alors à un rythme moins soutenu (prêt d'honneur et prêt professionnel notamment).

Si ces politiques attestent de la reconnaissance par le législateur d'un droit à l'emploi pour les veuves, elles n'en demeurent pas moins sexuées. Le contenu des emplois proposés aux veuves dans les écoles est, à cet égard, probant. Il s'agit soit de formations spécifiquement féminines liées le plus souvent aux travaux d'aiguille (couture, lingerie, bonneterie, gileterie, culotterie, dentellerie ou encore tricotage mécanique), soit d'emplois mixtes dans des secteurs traditionnels (papeterie-reliure, vannerie, paillage, rotinage, reliure, cartonnage, dorures) ; mais jamais de métiers traditionnellement masculins ${ }^{55}$. Une telle subversion a-t-elle existé dans le cadre des apprentissages chez un professionnel indépendant ? Leur mise en application, plus souple, a pu favoriser un tel phénomène. Mais cet empirisme est aussi la cause d'absence de données précises à leur propos, empêchant d'aboutir à toute conclusion fiable à ce sujet. À première vue, il ne semblerait pas que la discrimination constatée pour les écoles existe en matière de prêts d'honneur et de prêts professionnels, parce que les critères de leur attribution sont strictement identiques pour les veuves et les mutilés - à savoir leur honnêteté, leur besoin réel des sommes demandées et leur aptitude à exercer le métier envisagé. Or, ce dernier critère constitue en lui-même un levier de discrimination envers les veuves, parce qu'elles ne peuvent, en tant que femmes, être formées à tous les métiers ${ }^{56}$. C'est pourquoi l'on compte, parmi les veuves bénéficiaires d'un prêt d'honneur, une très forte proportion d'artisanes et de commerçantes dans les métiers du textile et du vêtement, secteur où elles peuvent certifier leur compétence ${ }^{57}$, et de très rares

54 Période pour laquelle les archives de l'ONMR livrent le plus de statistiques.

$55 \mathrm{ONMR}$, La rééducation professionnelle. Les écoles, les stations thermales, la rééducation chez le patron, les veuves de guerre, Paris, Imprimerie nationale, 1920 ; S.a., « La rééducation des mutilés.
Liste des écoles de rééducation », Journal des mutilés, 16 août 1924. p. 3.

56 Schweitzer Sylvie, Les femmes ont toujours travaillé. Une histoire de leurs métiers, $X I X^{e}-X X^{e}$ siècles,

Paris, Odile Jacob, 2002.

57 Sur les 734 prêts accordés, près de la moitié ont été attribués à des tricoteuses (152), à des couturières (71), à des bonnetières (16), à des lingères (15), à des confectionneuses (14), à des culottières (14), à des brodeuses (8), ou encore à des « ajoureuses », « chemisières », « corsetières », dentellières », « entrepreneuses en broderie », " gantières », 
«pâtissière ", « confiseuse ", « boulangère ", « charcutière ", « bouchère " ${ }^{\mathbf{8}}$, autant de métiers qui, à la différence des autres commerces alimentaires (tels que l'épicerie), nécessitent un apprentissage spécifique en complément de la pratique commerciale. Le temps conduit l'ONMR à prendre de plus en plus en considération des métiers du tertiaire qui, contrairement aux précédents,

constituent au lendemain de la guerre les emplois d'avenir pour les femmes. Les veuves aidées dans les années 1920 aux fins d'intégrer de tels métiers n'excèdent toutefois pas une trentaine (huit dactylographes, douze sténodactylographes, trois enseignantes, deux nourrices, une infirmière, une directrice de maison de santé, une dentiste et une chimiste).

Concernant l'attribution de machines à coudre, l'objet, en lui-même, dit tout. D'un usage exclusivement féminin, la machine à coudre est, dans la France de l'époque, un objet ordinaire des ménages français, notamment chez les plus modestes, où il remplit une double utilité domestique et professionnelle. Aux yeux d'Henri Chéron, président de l'ONMR et à l'initiative de cette politique, « la machine à coudre est indispensable au foyer où grandissent plusieurs enfants, surtout lorsque le foyer est privé de son chef. Rien ne peut être plus utile à une petite famille que la machine à coudre qui permet à la fois d'entretenir convenablement ses enfants et de se procurer les ressources complémentaires rendues indispensables par la pension allouée par l'État »59. Les critères d'attribution définis par l'ONMR confortent cette conception traditionnelle et familiale : les machines à coudre sont exclusivement dédiées aux veuves chargées de famille (les mutilés n'ont droit qu'à une machine à écrire en braille s'ils sont aveugles) avec une priorité accordée à celles qui résident dans les régions libérées. Les veuves remariées sont admises dès lors qu'elles ont de très nombreux enfants ${ }^{60}$. Cette politique rencontre un grand succès auprès des veuves de guerre, dont les demandes affluent. Environ 10 ooo d'entre elles sont satisfaites, parmi lesquelles celles des veuves de la France meurtrie du Nord-Est, de la France féconde de l'Ouest et de la France du textile du couloir rhodanien et du Pas-de-Calais ${ }^{61}$. Trois visages féminins somme toute très classiques et consensuels, mêlant la figure de la mère à celui de la victime (de la guerre ou du travail) et confortant un ordre social traditionnel dont la famille constitue la base et dans lequel la femme, travailleuse, reste à la maison.

Ainsi, les politiques de l'ONMR présentent le paradoxe de privilégier les veuves cheffes de famille sans leur donner la possibilité d'accéder à des formations ou à des activités susceptibles d'engendrer des revenus d'un chef de famille. Très féminines, les formations et les activités professionnelles soutenues par

« giletières », « piqueuse de bottine », «tisseuses »... (CAC, 200602052 et 3 , ONMR, procès-verbaux des séances du conseil de perfectionnement, 1918-1927).

58 On ne dénombre qu'une seule femme pour chacun de ces métiers.

59 CAC, 20050206 14, ONMR, conseil d'administration, assemblée plénière du 23 décembre 1920, rapport du conseil de perfectionnement par Constant Verlot, p. 66-67.

6o ONMR, L'attribution de machines à coudre aux veuves pensionnées de guerre, Paris, Imprimerie nationale, 1920.

6I CAC, ONMR, procès-verbal du conseil de perfectionnement, 1921-1926. 
l'ONMR ne leur permettent de prétendre qu'à des salaires d'appoint, pensés pour des épouses dont le revenu vient compléter celui de leur mari, plus conséquent ${ }^{62}$.

\section{Conclusion}

Le premier conflit mondial a provoqué un renouvellement important des institutions dédiées aux victimes de guerre. Presque inexistantes à la veille des hostilités, elles surgissent en nombre, dès 1914, pour secourir les familles éprouvées. On peut évaluer à un peu plus de 300 celles dédiées spécifiquement aux veuves et orphelins. Cette prise en charge sans précédent des veuves du point de vue quantitatif (celles-ci n'ont jamais eu autant d'institutions pour les aider) présente, qualitativement, une très forte continuité avec les œuvres caritatives du XIX ${ }^{\mathrm{e}}$ siècle. Le sort des veuves reste très fortement lié à celui des orphelin.e.s. Les veuves sont avant tout perçues comme des mères et maintenues dans une posture d'assistées. La création, en 1916 et 1917, des offices nationaux suscite un bouleversement de cette prise en considération. Les veuves, à l'instar des autres victimes de guerre, sont désormais considérées comme des « ayants droit », victimes qui, en raison du sacrifice consenti pour restaurer l'intégrité de la nation, ont des droits sur l'ensemble des Français. Ce changement de paradigme s'incarne dans le mode de fonctionnement des offices, gérés de manière paritaire. Les veuves, pour la première fois, participent à l'administration des institutions qui les prennent en charge. Par ailleurs, les politiques qui leur sont dédiées ne dépendent plus de la charge des orphelins mais sont élaborées et mises en application par l'ONMR, au même titre que celles consacrées aux mutilés. Reste que la nature et les critères d'attribution de ces politiques présentent une forte continuité idéologique avec ceux que prônent les œuvres de guerre. Très sexuées, ces politiques pensent les veuves encore et avant tout comme des mères de famille; les veuves sans enfant ne semblent pas exister aux yeux des responsables des offices.

Cette primauté de la famille sur la condition des femmes confirme le bilan nuancé de la Première Guerre mondiale en matière d'émancipation féminine. Mieux prises en compte, mieux considérées, les veuves de guerre restent, en dépit des innovations institutionnelles nées de la guerre, des victimes secondaires. Si ces bouleversements, à l'instar de la réforme des pensions, les ont fait passer du statut de veuve de combattant et mère d'orphelins à celui de cheffe de famille, ils n'ont pas provoqué leur reconnaissance individuelle, indépendamment de leur statut matrimonial ou de la taille de leur foyer. Ainsi, du point de vue des rapports de genre (analysés au prisme de la situation faite aux veuves de guerre), charité et solidarité n'apparaissent pas toujours antinomiques. Le processus d'étatisation de la protection sociale française, conforté par la Première Guerre mondiale, semble, en ce qui concerne la prise en compte des

62 Schweitzer Sylvie, Les femmes ont toujours travaillé... op. cit., p. III-II5; Menon Monique et
Silvera Rachel, « Salaires féminins: le point et l'appoint », dossier, Travail, Genre et Société, n 15 ,

2006/1, p. 27-138. 
femmes, avoir à court et moyen terme moins suscité de ruptures qu'entretenu

La prise en charge

des veuves

des continuités.

de querre

(France, 1914-1924)

Peggy Bette 